AperTO - Archivio Istituzionale Open Access dell'Università di Torino

\title{
Design and characterization of the beam monitor detectors of the Italian National Center of Oncological Hadron-therapy (CNAO)
}

\section{This is the author's manuscript}

Original Citation:

Availability:

This version is available http://hdl.handle.net/2318/128978

since 2016-06-28T18:39:02Z

Published version:

DOI:10.1016/j.nima.2012.10.004

Terms of use:

Open Access

Anyone can freely access the full text of works made available as "Open Access". Works made available under a Creative Commons license can be used according to the terms and conditions of said license. Use of all other works requires consent of the right holder (author or publisher) if not exempted from copyright protection by the applicable law. 


\section{(2) \\ UNIVERSITÀ DEGLI STUDI DI TORINO}

This Accepted Author Manuscript (AAM) is copyrighted and published by Elsevier. It is posted here by agreement between Elsevier and the University of Turin. Changes resulting from the publishing process - such as editing, corrections, structural formatting, and other quality control mechanisms may not be reflected in this version of the text. The definitive version of the text was subsequently published in Nuclear Instruments and Methods in Physics Research A 698 (2013) 202-207

http://dx.doi.org/10.1016/j.nima.2012.10.004

You may download, copy and otherwise use the AAM for non-commercial purposes provided that your license is limited by the following restrictions:

(1) You may use this AAM for non-commercial purposes only under the terms of the CC-BY-NC-ND license.

(2) The integrity of the work and identification of the author, copyright owner, and publisher must be preserved in any copy.

(3) You must attribute this AAM in the following format: Creative Commons BY-NC-ND license (http://creativecommons.org/licenses/by-nc-nd/4.0/deed.en), [+ Digital Object Identifier link to the published journal article on Elsevier's ScienceDirect ${ }^{\circledR}$ platform] 


\title{
Design and characterization of the beam monitor detectors of the Italian National Center of Oncological Hadron-therapy (CNAO)
}

\author{
S. Giordanengo ${ }^{1}$, M. Donetti ${ }^{1,3}$, M. A. Garella ${ }^{1,3}$, F. Marchetto ${ }^{1}$, G. Alampi ${ }^{1}$, A. Ansarinejad ${ }^{4}$, V. Monaco ${ }^{1,2}$, \\ M.Mucchi ${ }^{1}$, I. A. Pecka ${ }^{5}$, C. Peroni ${ }^{1,2}$, R. Sacchi ${ }^{1,2}$, M. Scalise ${ }^{1}$, C. Tomba ${ }^{6}$, R. Cirio ${ }^{1,2}$ \\ ${ }^{1}$ Istituto Nazionale di Fisica Nucleare (INFN), via P. Giuria 1, 10125 Torino, Italy \\ ${ }^{2}$ Dipartimento di Fisica, Universita' di Torino, via P. Giuria 1, 10125 Torino, Italy \\ ${ }^{3}$ Fondazione CNAO, strada Campeggi, 27100 Pavia, Italy \\ ${ }^{4}$ presently at the Nuclear Science Research School, Nuclear Science and Technology Research Institute, \\ Tehran - Iran \\ ${ }^{5}$ presently at Kantonsspital Luzern, Zurich Area, Switzerland \\ ${ }^{6}$ presently at Institut Néel/CNRS-Université Joseph Fourier, 25 rue des Martyrs, 38042 Grenoble, France
}

\begin{abstract}
A new hadron-therapy facility implementing an active beam scanning technique has been developed at the italian National Center of Oncological Hadron-therapy (CNAO). This paper presents the design and the characterization of the beam monitor detectors developed for the online monitoring and control of the dose delivered during a treatment at CNAO. The detectors are based on five parallel-plate transmission ionization chambers with either a single large electrode or electrodes segmented in 128 strips (strip chambers) and 32x32 pixels (pixel chamber). The detectors are arranged in two independent boxes with an active area larger than $200 \mathrm{x} 200 \mathrm{~mm}^{2}$ and a total water equivalent thickness along the beam path of about $0.9 \mathrm{~mm}$. A custom front-end chip with 64 channels converts the integrated ionization channels without dead-time.

The detectors were tested at the clinical proton beam facility of the Paul Scherrer Institut (PSI) which implements a spot scanning technique, each spot being characterized by a predefined number of protons delivered with a pencil beam in a specified point of the irradiation field. The short-term instability was measured by delivering several identical spots in a time interval of few tenths of seconds and is found to be lower than $0.3 \%$. The non-uniformity, measured by delivering sequences of spots in different points of the detector surface, results to be lower than $1 \%$ in the single electrode chambers and lower than $1.5 \%$ in the strip and pixel chambers, reducing to less than $0.5 \%$ and $1 \%$ respectively in the restricted $100 \times 100 \mathrm{~mm}^{2}$ central area of the detector.
\end{abstract}

Keywords: hadron-therapy; beam delivery system; on-line detectors; ionization chamber 


\section{Introduction}

The Italian National Center of Oncological Hadron-therapy (CNAO), located in Pavia (Italy) [1], is one of the few facilities in the world designed for treating tumors with protons [2] and carbon ions [3] beams. It implements an active scanning technique where a pencil beam irradiates the tumour with a large number of spots, each spot being characterized by the beam energy, the number of particles and the position in the transverse plane. Such irradiation method requires a precise, fast, stable and uniform beam monitoring system allowing the on-line measurement of the number of particles delivered in the spot and of the spot position in the irradiation field.

The first part of the paper describes the beam monitoring detectors, developed by CNAO and by the group of the Istituto Nazionale di Fisica Nucleare and the University of Torino, which are currently installed in the four treatment lines of CNAO. Prior to the installation, a set of detectors was tested at the Paul Scherrer Institut (PSI) in Villigen (CH) with a clinical proton beam [4] delivered with a spot scanning technique. These tests allowed determining the shortterm stability and the uniformity of the detectors in an irradiation environment similar to CNAO. The results are reported in the second part of this paper.

\section{Detector description}

The detectors developed for CNAO are based on parallel-plate ionization chambers with either a single large electrode or electrodes segmented in strips or pixels. Their design is similar to other detectors successfully developed by the Torino group for different medical applications $[5,6]$. For safety and redundancy requirements, two independent detectors have been designed for each treatment line: they are housed in separate boxes (called BOX1 and BOX2) and are read out and processed independently during the treatment. BOX1 contains an integral chamber (INT1) with a large area anode for the measurement of the beam current followed by two chambers where the anode is segmented in strips (STPX and STPY) proceeding the measurement of the beam position along orthogonal directions. BOX2 contains another integral chamber (INT2) followed by a chamber where the anode is segmented in pixels (PXC).

A schematic drawing of the detector assemblies is shown in figure 1. All the cathodes are made by a $12 \mu \mathrm{m}$ thick mylar foil with an aluminum layer of few tens of $\mathrm{nm}$ on one side with the exception of the common cathode of the two strip chambers which is twice as thick and aluminized on both sides. The anodes of the integral and strip chambers are made by a $25 \mu \mathrm{m}$ thick kapton foil covered by a $17 \mu \mathrm{m}$ aluminum layer engraved with standard PCB technique to obtain 128 conductive strips $1.55 \mathrm{~mm}$ wide with a pitch of $1.65 \mathrm{~mm}$. The pixel anode consists of a $50 \mu \mathrm{m}$ thick kapton layer on which a $20 \mu \mathrm{m}$ thick film of copper has been deposited on both sides distributed in a $32 \times 32$ matrix of pixels on one side and tracks, one for each pixel, on the other side, the connection being achieved through a conductive hole. The area of each 
conductive pixel is $6.5 \times 6.5 \mathrm{~mm}^{2}$ and the pitch is $6.6 \mathrm{~mm}$. To avoid charge buildup and leakage currents, a guard ring is positioned around the sensitive areas of the anodes and set at the anode potential.
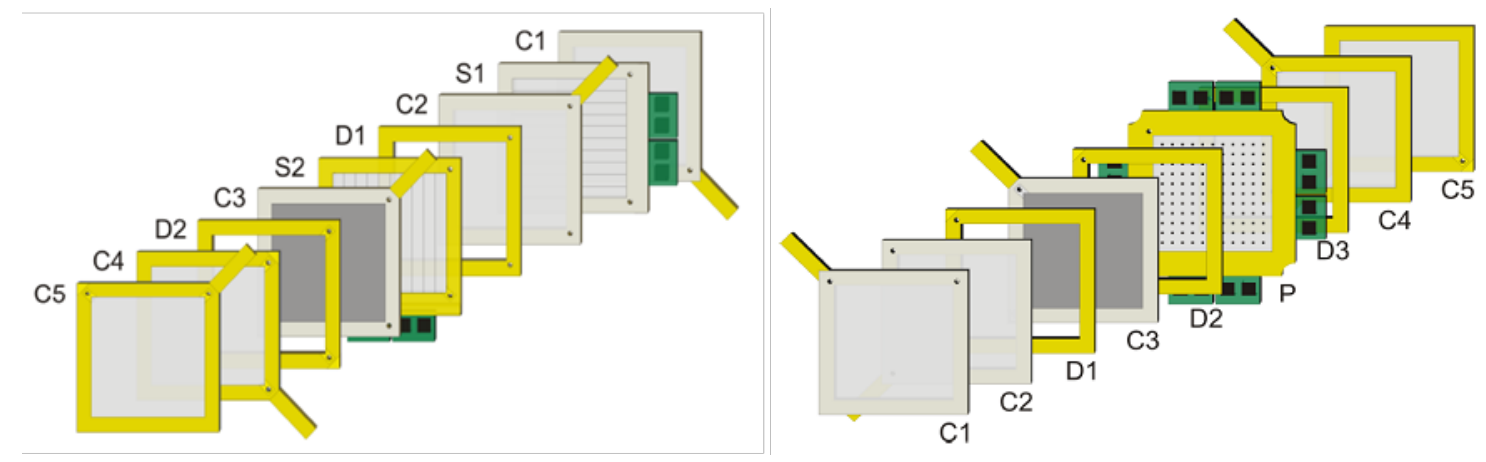

Figure 1: schematic drawing of the detector assembly of BOX1 (left) and BOX2 (right): S1 and S2 are the anodes segmented in strips, $\mathrm{P}$ is the anode segmented in pixels, C3 is the anode of the integral chambers, $\mathrm{C} 2$ and $\mathrm{C} 4$ are the cathodes. The frames D1, D2 and D3 are needed to provide the desired gap between electrodes while $\mathrm{C} 1$ and $\mathrm{C} 5$ are added to provide an additional gas gap on both sides of the assembly, as explained in the text.

As described in [5], the electrodes are stretched and glued on fiberglass frames which also provide mechanical support for the front-end readout boards. Additional frames are added between the electrodes to provide a gas gap of $5 \mathrm{~mm}$ for each chamber. To fill the chamber with a gas an overpressure inside the frame package is required. The combination of the rather large area and thin foils can result in a considerable change of the gas gap. To prevent this effect, two blank chambers are added, one on each side of the active chambers, where the gas flows at the same pressure. The blank chambers have been made with a frame supporting a $12 \mu \mathrm{m}$ thick aluminized mylar foil. In order to reduce the induced electrical noise these foils are set to ground. A gas fitting on each of the blank chamber provides the in and the out gaslet. Finally, the gas path through the active chamber was constrained via grooves and holes machined on the frames.

The frames are stack on top of each other and are aligned by means of high precision mechanical pins, as shown in figure 2.

The external box enclosure of the box is sealed in order to operate the chambers with gases; in the front and the back covers, two windows made of a thin aluminized mylar foil are used to 
ensure gas tightness minimizing the material along the beam path. Outside of the gas region the box houses a slow control board, used to provide the operating voltages and to measure the temperature and the pressure in the gas region, and an interface board for the data acquisition.

The size of each box is $50 \times 70 \times 10 \mathrm{~cm}^{3}$. The total sensitive area is $240 \times 240 \mathrm{~mm}^{2}$ for the integral chambers and $211 \times 211 \mathrm{~mm}^{2}$ for the strip and pixel chambers and the total water equivalent thickness along the beam path is $0.9 \mathrm{~mm}$. In order to maximize the collection efficiency, the chambers are operated with $\mathrm{N}_{2}$ and with a electric field of about $800 \mathrm{~V} / \mathrm{cm}$, generated by polarizing the cathodes to $-400 \mathrm{~V}$ and the anodes at the reference voltage of the front-end input $(2.05 \mathrm{~V})$. It was observed that in these operating conditions the collection efficiency is about $100 \%[6]$.

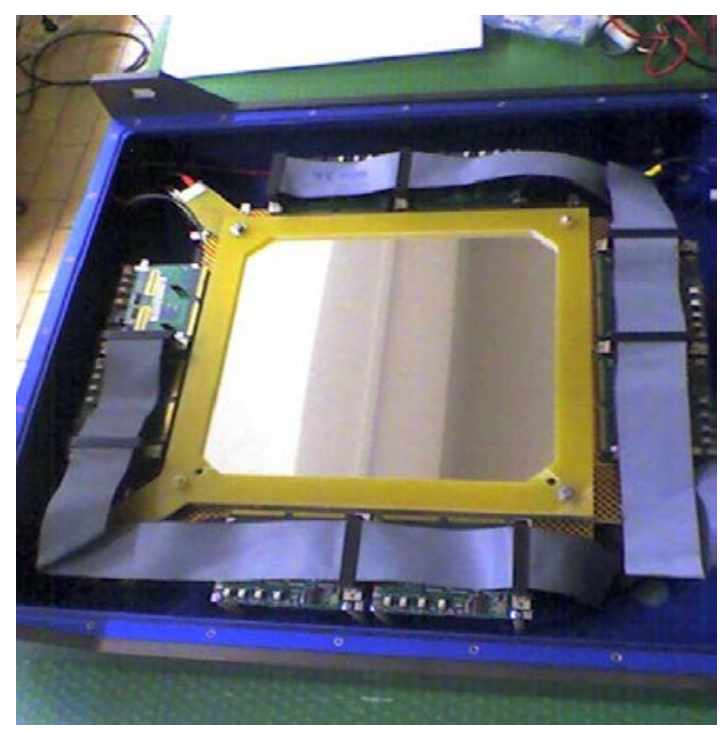

Figure 2: picture of BOX2. The front cover has been removed to show the inner assembly.

The read-out electronics of the detector is based on two versions of a 64-channels wide dynamic range current-to-frequency converter ASIC developed by the Torino group: the TERA06 [7, 8] for the strip and pixel chambers and the TERA07 [9] for the integral chambers. The main features of the two versions are summarized in table 1. Their design is based on the recycling integrator architecture [10]: the input currents from each channel are integrated and the numbers of pulses proportional to the collected charge are sent to 64 synchronous counters. The charge corresponding to a pulse (charge quantum) can be set externally; for the current application it is set to $200 \mathrm{fC}$. All the counters can be latched simultaneously and the contents stored in registers which can be individually addressed and multiplexed to the output bus. Such design is intrinsically dead-time free as the latch operation does not affect the counting itself. In each detector, data from the integral chambers and from the strip and pixel chambers are acquired 
independently. At CNAO the integral chambers are read out at a frequency of $1 \mathrm{MHz}$ whereas the readout frequency of strip and pixel chambers is about $10 \mathrm{kHz}$.

\begin{tabular}{l|ll}
\hline & TERAO6 & TERAO7 \\
\hline Technology & CMOS $0.8 \mu \mathrm{m}$ & CMOS $0.35 \mu \mathrm{m}$ \\
Die Size & $6 \times 7 \mathrm{~mm}^{2}$ & $5.4 \times 4.5 \mathrm{~mm}^{2}$ \\
Number of channels & 64 & 64 \\
Input current & unipolar & bipolar \\
Charge quantum range & $100 \mathrm{fC}-800 \mathrm{fC}$ & $25 \mathrm{fC}-1.155 \mathrm{pC}$ \\
Max count frequency & $5 \mathrm{MHz}$ & $20 \mathrm{MHz}$ \\
Max input current * & $1 \mu \mathrm{A}$ & $4 \mu \mathrm{A}$ \\
Non linearity * & $<1 \%$ & $<1.2 \%$ \\
Average dark noise * & $1.1 \mathrm{~Hz}$ & $1.3 \mathrm{~Hz}$ \\
Charge quantum spread * & $1.3 \%$ & $1.3 \%$ \\
\hline
\end{tabular}

Table 1: main features of the TERA chips (an asterisk is used to indicate the quantities that are specific of a charge quantum of $200 \mathrm{fC}$ ).

\section{Test set-up}

The test of the detectors was performed at the Paul Scherrer Institut (PSI) in Villigen (CH) with the clinical proton beam of the isocentric scanning gantry 1 [11]. Here, the dose is delivered during the treatment as a time sequence of individual pencil beam depositions, called spots, the 
number of protons in each spot being monitored by a set of parallel plane ionization chambers. When the desired number of protons has been reached for a given spot, the beam is switched off with a kicker magnet and the beam position is changed. The time per spot varies typically from 0.5 to $100 \mathrm{~ms}$ [12]. The calibration of the monitors from units of electric charge to number of incident protons is provided by Faraday-cup measurements. The beam has a Gaussian shape with 7-8 $\mathrm{mm}$ FWHM and a scanning step of $5 \mathrm{~mm}$.

For this test, a $177 \mathrm{MeV}$ proton beam with an average intensity of $4 \cdot 10^{8}$ protons per second was delivered. The gantry was rotated at $90^{\circ}$ and the two detectors positioned facing each other on the patient table, perpendicularly to the beam, at a distance of about $0.5 \mathrm{~m}$ from the nozzle (figure 3 ).

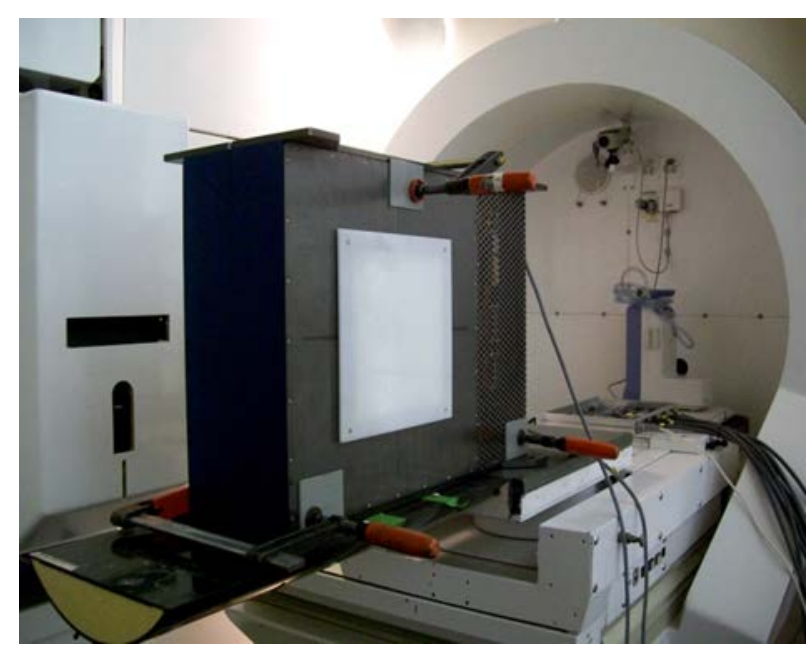

Figure 3: picture of the test setup into the PSI gantry 1 room.

The shift of the beam along the horizontal $\mathrm{X}$ axis was achieved by the action of a sweeper magnet while the shift along the vertical Y axis was applied by moving the patient table [3]. The detector was operated at the nominal high voltage setting $(-400 \mathrm{~V})$ and the charge quantum was set to 200 fC.

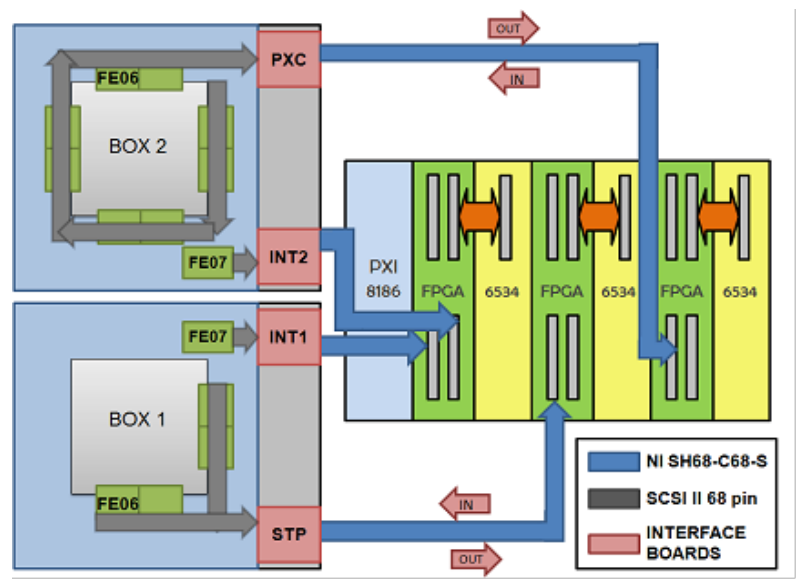


Figure 4: layout of the data acquisition system. The three FPGA are used to acquire monitor data

The data acquisition system was based on the National Instruments PXI platform and was similar to the system developed for CNAO; a PXI crate containing three FPGA boards (NI PXI-7811R), each connected to a digital DAQ board (NI PXI-6534), and a system controller (NI PXI-8186). The layout is shown in figure 4.

The software was designed to readout the five chambers at the same time, either with a fixed frequency of $2 \mathrm{kHz}$ or synchronized in time with the spot delivery by using the signal from the PSI kicker magnet. At each readout, the number of counts of each detector channel was stored on file as an unsigned 16 bit integer for later analysis.

\section{Results}

\subsection{Background current}

In the design of the chambers, special care was devoted in limiting the dark background current as it may affect the dose measurements, especially when low beam currents are used. To achieve the prescribed dose accuracy of $\pm 2.5 \%$ [1], a dark current lower than $1 \%$ of the signal is required during the clinical application.

Before irradiation, the background currents were determined by taking several measurements at regular time intervals of 60 s and by averaging the results. As an example, the average number of counts per second measured for each channel of the pixel chamber is shown in figure 5 .

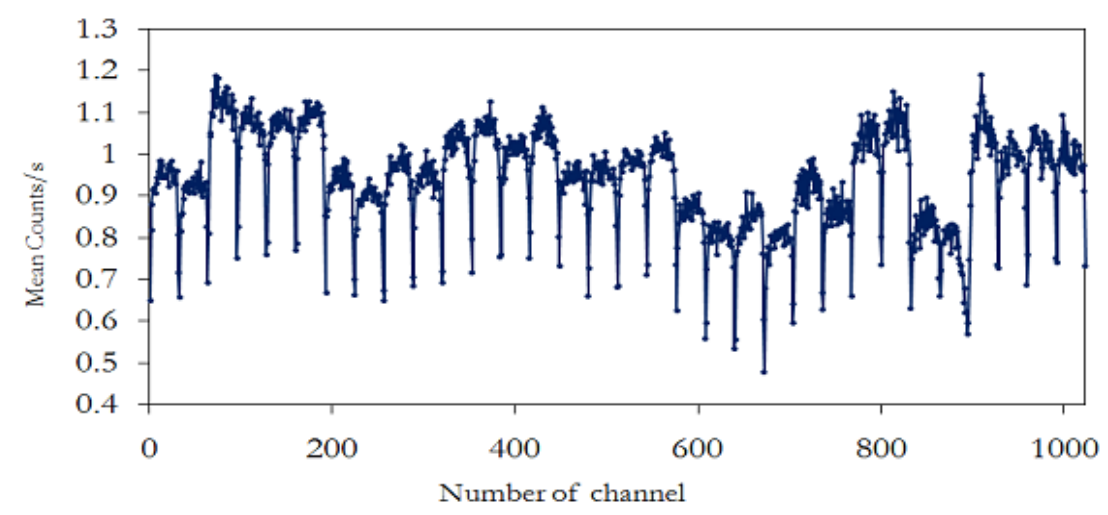

Figure 5: average number of counts per second for the 1024 channels of the pixel chamber. 
The regular structure corresponding to groups of 64 channels connected to the same TERA chip was already observed in [8]. The average value of 0.94 counts/s corresponds to an average background current per channel of $190 \mathrm{fA}$; similarly, background currents of $170 \mathrm{fA},-210 \mathrm{fA}$ and $190 \mathrm{fA}$ were observed in the STP, INT1 and INT2 chambers respectively. Small or no dependence of the background on the high voltage was observed in the range $100 /-600 \mathrm{~V}$, indicating that the largest background contribution comes from the front-end electronics.

Considering that the typical currents during a treatment are of the order of $10 \mathrm{nA}$, the measured background contribution is well within requirements and can be safely neglected.

\subsection{Short-term stability}

The beam monitor performance must be stable during the irradiations. To fulfil the clinical requirements, the short-term stability under identical irradiation condition should be better than $1 \%$.

The short-term stability as a function of time was tested by delivering several sequences of 400 identical spots in the same central position of the monitor chambers. Each spot was required to contain $7.8 \times 10^{7}$ protons, corresponding to a spot duration of about 20 ms followed by a $12 \mathrm{~ms}$ interval before the following spot, for a total test-time of $12 \mathrm{~s}$.

Figure 6 shows the number of counts for each spot as measured by the two integral chambers. A $5 \%$ difference between the gains of the two chambers is observed which may originate both from different gains of the TERA chips and from mechanical tolerances. However, this difference has no impact as the absolute calibration of each integral chamber is regularly performed at CNAO. It can be seen from the figure that fluctuations of the number of counts for both chambers are limited within $\pm 0.5 \%$ of the average with an RMS of $0.3 \%$ for both INT1 and INT2. 

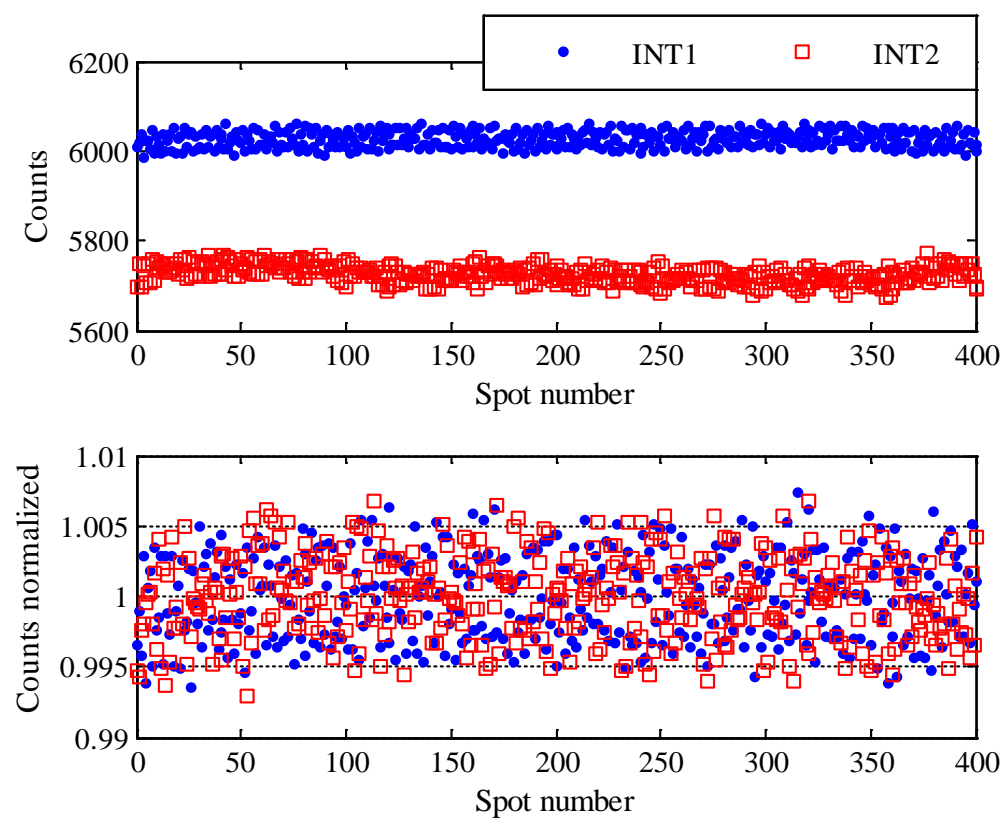

Figure 6: number of counts as a function of the spot number for the integral chambers (top) and the same quantity normalized to the average (bottom). The dashed lines correspond to the $\pm 0.5 \%$ deviation from the average.

The RMS of the distribution of the ratio of counts of INT1 and INT2 is found to be $0.4 \%$, suggesting that the fluctuations observed in the two chambers are effects not correlated with the beam variations.

Similar results are obtained using strip and pixel chambers, where the total number of counts is defined by adding the counts of 80 channels (10x10 pixels) centered on the strip (pixel) with the maximum number of counts. Limiting the sum to a restricted number of channels, the influence of the noise is reduced still ensuring that the beam tails are properly considered. The results are summarized in table 2.

\begin{tabular}{l|llcll}
\hline & INT1 & INT2 & STPX & STPY & PXC \\
\hline max-min (\%) & 1.4 & 1.4 & 0.62 & 0.89 & 0.77 \\
RMS (\%) & 0.30 & 0.29 & 0.11 & 0.14 & 0.12 \\
\hline
\end{tabular}


Table 2: short-term stability of all the chambers measured in a run with 400 identical spots. Both the maximum fluctuation and the RMS are reported, expressed as the percentage of the average.

\subsection{Spatial resolution}

To fulfil the required precision for beam delivering, each spot position error should be lower than $1 \mathrm{~mm}$, implying that a beam monitor position resolution better than $0.5 \mathrm{~mm}$ should be achieved.

Figure 7 shows the beam position and the width FWHM of the beam profile, measured using both strip and pixel chambers, in the same run discussed above. For the position and the width the following formulae are used

$$
\begin{gathered}
X_{\text {beam }}=\frac{\sum_{i} x_{i} n_{i}}{N} \\
\sigma_{\tilde{X}}^{F W H M}=2.35 \sqrt{\frac{1}{N} \sum_{i} n_{i}\left(x_{i}-X_{\text {beam }}\right)^{2}}
\end{gathered}
$$

and similarly for $Y$, where $x_{i}$ is the position of strip $i, n_{i}$ the corresponding number of counts and $N=\sum_{i} n_{i}$. 


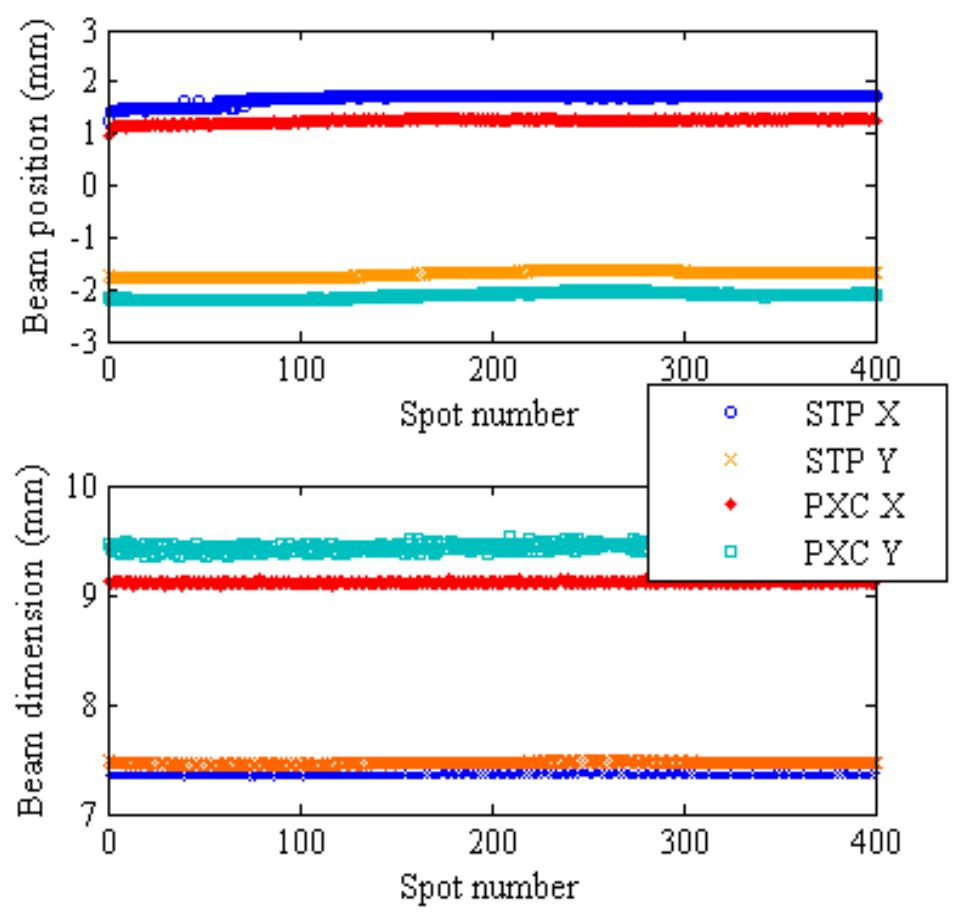

Figure 7: beam position and beam FWHM measured with the strip and pixel chambers in a run with 400 identical spots.

In these formulae, the strips are defined in the pixel chamber as the sum of the counts of all the pixels corresponding to the same column or row, depending on the desired orientation. Also, to reduce the effect of noise, the sum is extended over the 11 (4) channels centered on the strip (pixel) corresponding to the maximum.

The positions measured by the strip and pixel chambers are very similar, the small difference being caused by a non perfect alignment of the two detector boxes relative to the beam. On the contrary, the beam FWHM measured by the pixel chamber appears to be largely overestimated because of the large pixel size, comparable to the width of the beam. The RMS of the position distribution is dominated by the detector resolution and is found to be $0.091 \mathrm{~mm}$ and $0.074 \mathrm{~mm}$ for STPX and STPY chambers and $0.18 \mathrm{~mm}$ for the PXC chamber, well below the clinical requirements. It should be noted however that the resolution is dependent on the number of protons delivered in each spot and is expected to increase by a factor of about 3 if the number of protons delivered in the spot is reduced by a factor 10 . 


\subsection{Uniformity}

Beam monitors have to guarantee that the defined volume will receive a uniform amount of dose. Any measurements dependence, such as beam position dependence, has to be limited to $1 \%$ in order to avoid systematic over or under-dose.

The uniformity of the chambers was first tested by moving the beam horizontally, acting on the sweeper magnet. A sequence consisting of 37 spots spaced by $5 \mathrm{~mm}$ along a horizontal line, from $X=+90 \mathrm{~mm}$ to $X=-90 \mathrm{~mm}$, followed by the same sequence of 37 spots delivered in a reversed order, was repeated three times. Each spot was required to contain $2.4 \times 10^{8}$ protons. As a result, six identical overlapping spots were delivered in each of the 37 points. The stability, measured as the RMS of the number of counts between the six measurements, was found to be in each point below $0.2 \%$ in the integral ${ }^{1}$ and strip chambers and at most $0.4 \%$ in a few points of the pixel chamber, in rough agreement with the results of table 2 . The non-uniformity of the chambers is shown in figure 8 where the average number of counts in each point, normalized to the average number of counts for all the points, is shown as a function of $X$. It is found to be less than $\pm 1 \%$ for the INT and STPY chambers and less than $\pm 1.5 \%$ for the STPX and PXC chamber. It should be noted that the uniformity of the strip and pixel chambers is affected by differences of the electronic gain of the channels and could be substantially improved by applying channel-bychannel calibration coefficients [13].

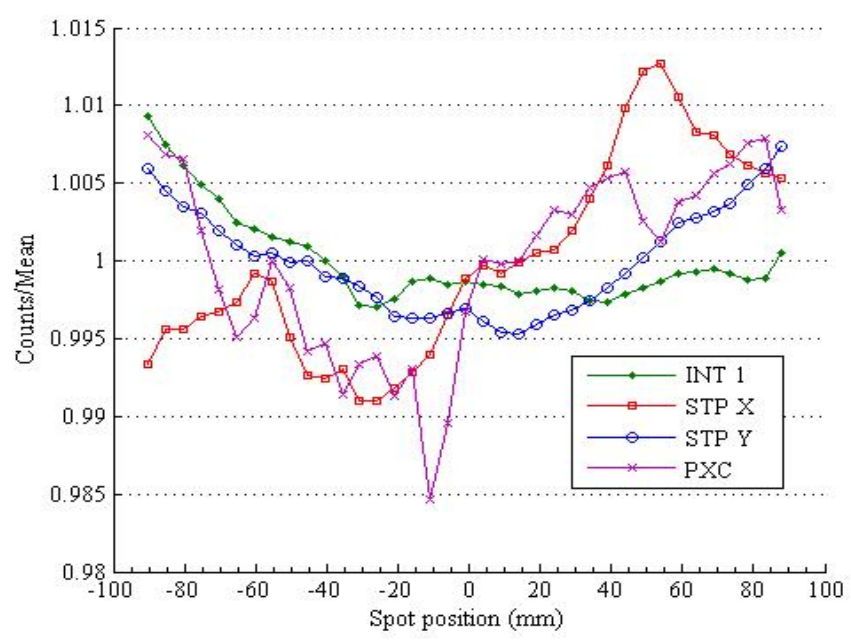

Figure 8: uniformity of the chambers as a function of the $X$ position along the horizontal central line. The average number of counts in each point, normalized to the average over all the 37 points, is shown.

\footnotetext{
${ }^{1}$ For technical problems only data from one of the two integral chambers were available for this analysis.
} 
Finally, the uniformity was tested over a square central area of $100 \times 100 \mathrm{~mm}^{2}$ by delivering a grid of $21 \times 21$ spots spaced by $5 \mathrm{~mm}$ and containing $6 \times 10^{7}$ protons each. The sequence was repeated twice and the average number of counts was calculated in each point of the grid. The nonuniformity is shown in figure 9 where the number of counts in each point of the grid, normalized to the average number of counts for all the points, is shown for the two integral chambers. The results, summarized in table 3 , show that a non-uniformity as small as $0.5 \%$ is achieved in the central region of the integral chambers, which increases to about $1 \%$ for the strip an pixel chambers.
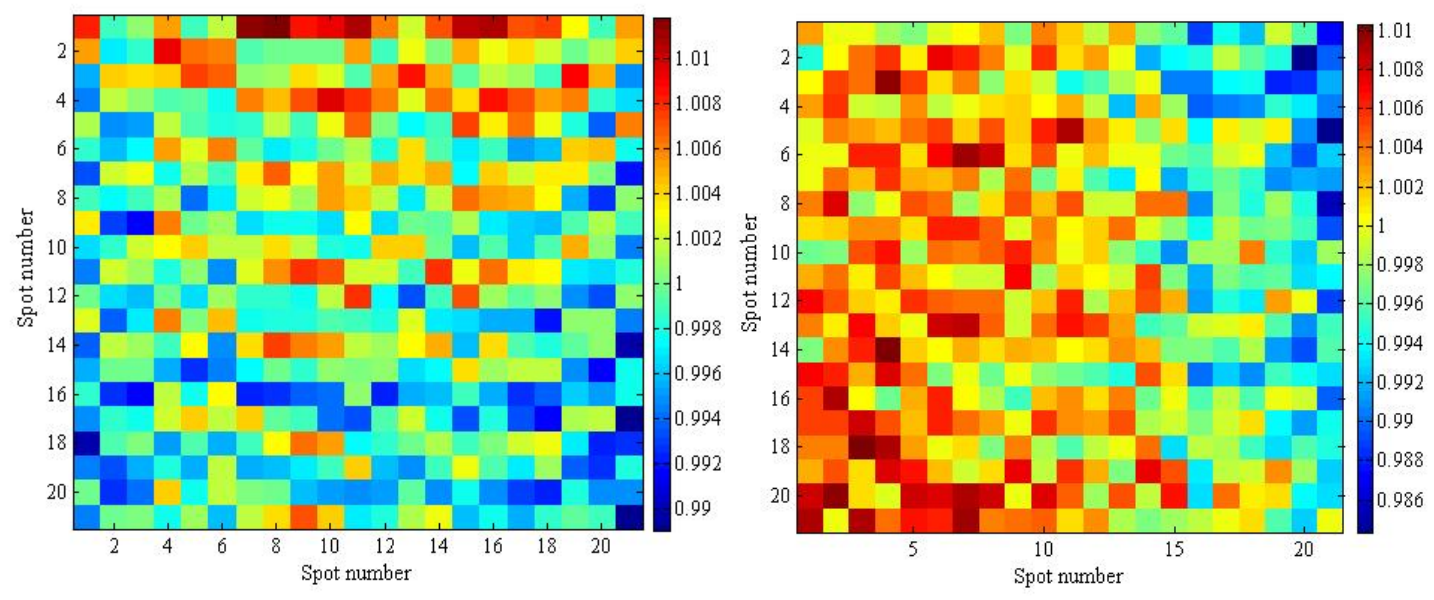

Figure 9: uniformity of the two integral chambers measured in a $21 \times 21$ grid of points covering an area of $100 \times 100 \mathrm{~mm}^{2}$. The number of counts in each point of the grid normalized to the average number of counts over all the points of the grid, is shown.

\begin{tabular}{l|lllll}
\hline & INT1 & INT2 & STPX & STPY & PXC \\
\hline max-min (\%) & 2.3 & 2.6 & 3.7 & 1.8 & 5.7 \\
RMS (\%) & 0.434 & 0.504 & 0.849 & 0.399 & 1.120 \\
\hline
\end{tabular}

Table 3: non-uniformity of the chambers over a central area of $100 \times 100 \mathrm{~mm}^{2}$. Both the maximum fluctuation and the RMS over a $21 \times 21$ grid of points are reported, expressed as the percentage of the average. 


\section{Conclusions}

Two detectors based on parallel-plate transmission ionization chambers have been developed for monitoring the beam in each of the treatment lines of the Italian Centro Nazionale di Adroterapia Oncologica (CNAO). The first detector contains an integral chamber with a single large electrode and two chambers with the electrodes segmented in strips; the second detector contains an identical integral chamber and a second chamber with the electrode segmented in pixels. Both detectors were tested at the clinical proton beam facility of the Paul Scherrer Institut (PSI), the aim of the test being to determine the uniformity and the short-term stability of the detectors.

The results can be summarized as follows:

- the background currents of the channels are all within $\pm 250 \mathrm{fA}$;

- the short-term stability of all the chambers results better than $0.3 \%$;

- the beam position resolution, for spots with more than $7 \times 10^{7}$ protons, is better than 0.1 $\mathrm{mm}$ with the strip chambers and $0.2 \mathrm{~mm}$ with the pixel chamber;

- the non-uniformity of the integral chambers is about $1 \%$, reducing to $0.5 \%$ if only the central area is considered. Larger non-uniformities of strip and pixel chambers are observed which can be improved by applying channel-by-channel calibration coefficients.

These results are all within the CNAO design tolerances of maximum background current, short term instability and non-uniformity and beam position resolution of $100 \mathrm{pA}, \pm 1 \%$ and $\pm 0.5 \mathrm{~mm}$ respectively.

The detectors are currently successfully used at CNAO for the monitoring of the proton beam during the treatments of the first patients.

\section{Acknowledgements}

We would like to thank Dr. Eros Pedroni, Dr. Shixion Lin and all the PSI proton-therapy group for giving us the opportunity to access the PSI facility and for helping during the measurements. 


\section{References}

[1] S. Rossi et al., Eur. Phys. J. Plus (2011) 126.

[2] A. R. Smith et al., Med. Phys. 36 (2009) 556.

[3] O. Jackel et al., Med. Phys. 35 (2008) 5653.

[4] E. Pedroni et al., Med. Phys. 22 (1995) 37.

[5] A. LaRosa et al., Nucl. Instr. and Meth. A 565 (2006) 833.

[6] N. Givechi et al., Nucl. Instr. and Meth. A 572 (2007) 1094.

[7] G. Mazza, et al., IEEE Trans. Nucl. Sci. NS-52 (4) (2005) 847.

[8] A. LaRosa et al., Nucl. Instr. and Meth. A 586 (2008) 270.

[9] A. LaRosa et al., Nucl. Instr. and Meth. A 583 (2007) 461.

[10] B. Gottschalk et al., Nucl. Instr. and Meth. A 207 (1983) 417.

[11] E. Pedroni et al., Phys. Med. Biol. 50 (2005) 541.

[12] S. Lin et al., Med. Phys. 36 (2009) 5331.

[13] R. Bonin et al., Nucl. Instr. and Meth. A 519 (2004) 674. 